\title{
Erratum: Third-order nonlinearity of graphene: Effects of phenomenological relaxation and finite temperature [Phys. Rev. B 91, 235320 (2015)]
}

\author{
J. L. Cheng, N. Vermeulen, and J. E. Sipe \\ (Received 22 December 2015; published 7 January 2016)
}

DOI: 10.1103/PhysRevB.93.039904

We have become aware (see the discussion in Ref. [1]) of a sign error in the third term in Eq. (25). The correct equation reads

$$
\mathcal{S}_{7}\left(\vartheta, \vartheta_{0}\right)=\mathcal{H}_{\mu}\left(\vartheta_{0}\right)\left(\frac{A_{2}}{\vartheta_{1}^{2}}-\frac{A_{3}}{\vartheta_{0} \vartheta_{1}}\right)+\mathcal{H}_{\mu}(\vartheta)\left(\frac{A_{3}}{\vartheta \vartheta_{1}}-\frac{A_{2}}{\vartheta_{1}^{2}}\right)-\mathcal{I}_{\mu}(\vartheta) \frac{A_{2}}{\vartheta_{1}}+\frac{1}{|\mu|} \frac{A_{3}}{\vartheta \vartheta_{0}},
$$

The error is purely typographical. All the results and discussions in the paper are based on the correct equation.

[1] S. A. Mikhailov, Quantum theory of the third-order nonlinear electrodynamic effects of graphene, arXiv:1506.00534. 